\title{
The fitness cost of mismatch repair mutators in Saccharomyces cerevisiae: partitioning the mutational load
}

\author{
Benjamin Galeota-Sprung ${ }^{1} \cdot$ Breanna Guindon ${ }^{1} \cdot$ Paul Sniegowski ${ }^{1}$
}

Received: 26 April 2019 / Revised: 30 July 2019 / Accepted: 12 August 2019 / Published online: 12 September 2019

(c) The Author(s), under exclusive licence to The Genetics Society 2019

\begin{abstract}
Mutational load is the depression in a population's mean fitness that results from the continual influx of deleterious mutations. Here, we directly estimate the mutational load in a population of haploid Saccharomyces cerevisiae that are deficient for mismatch repair. We partition the load in haploids into two components. To estimate the load due to nonlethal mutations, we measure the competitive fitness of hundreds of randomly selected clones from both mismatch-repair-deficient and -proficient populations. Computation of the mean clone fitness for the mismatch-repair-deficient strain permits an estimation of the nonlethal load, and the histogram of fitness provides an interesting visualization of a loaded population. In a separate experiment, in order to estimate the load due to lethal mutations (i.e. the lethal mutation rate), we manipulate thousands of individual pairs of mother and daughter cells and track their fates. These two approaches yield point estimates for the two contributors to load, and the addition of these estimates is nearly equal to the separately measured short-term competitive fitness deficit for the mismatch-repair-deficient strain. This correspondence suggests that there is no need to invoke direct fitness effects to explain the fitness difference between mismatch-repair-deficient and -proficient strains. Assays in diploids are consistent with deleterious mutations in diploids tending towards recessivity. These results enhance our understanding of mutational load, a central population genetics concept, and we discuss their implications for the evolution of mutation rates.
\end{abstract}

\section{Introduction}

An evolving population experiences a continual influx of mutations, the vast majority of which, excluding neutral mutations, are likely to be deleterious (Fisher 1930). A deleterious allele in a haploid population will attain an equilibrium frequency that is the quotient of the mutation rate to that allele and the selection coefficient against it (Danforth 1923). The influx of deleterious mutations causes a depression in the population's mean fitness that is termed the mutational load (Muller 1950), and the load at equilibrium is equal to the deleterious mutation rate (Haldane 1937). Because all populations experience mutation, all

Supplementary information The online version of this article (https:// doi.org/10.1038/s41437-019-0267-2) contains supplementary material, which is available to authorized users.

Benjamin Galeota-Sprung

bsprung@gmail.com

1 University of Pennsylvania, Philadelphia, PA, USA populations experience load, and a substantial proportion of the genetic variance for fitness in natural populations is due to mutational load (Charlesworth 2015).

Mutational load is closely connected to the evolution of mutation rates. Consider an asexual population in which there is genetic variation for the mutation rate: within such a population, distinct lineages with differing mutation rates experience differing loads and therefore possess differing mean fitnesses. In this way a downward selective pressure on the mutation rate is realized. This pressure is indirect in the sense that modifiers of the mutation rate are subject to selection without affecting any physiological property immediately related to fitness. The existence of ancient and highly conserved systems for replication fidelity (including proofreading, mismatch repair, and nucleotide excision repair) attests to the persistence of this selective pressure (Raynes and Sniegowski 2014).

In evolving populations, lineages with higher mutation rates ("mutators") are continually produced by mutation to any of numerous mutation-rate-affecting loci. In the absence of beneficial mutations, the expected frequency of mutators within a population depends on the increase in the 
deleterious mutation rate caused by the mutator allele, the rate of mutation from wild type to mutator, and the mean selective effect of newly arising deleterious mutations (Johnson 1999; Desai and Fisher 2011). Investigations of natural and clinical isolates of Escherichia coli and other bacteria have shown that mutators of one to two orders of magnitude in strength, often defective in mismatch repair, are present at low but notable frequencies in many populations (Jyssum 1960; Gross and Siegel 1981; LeClerc et al. 1996; Matic et al. 1997; Oliver et al. 2000; Denamur et al. 2002; Richardson et al. 2002; Trong et al. 2005; Denamur and Matic 2006; Gould et al. 2007; reviewed in Raynes and Sniegowski 2014). Evolution experiments conducted with $E$. coli have demonstrated that mutators can displace wild types by virtue of their increased access to beneficial mutations (Cox and Gibson 1974; Chao and Cox 1983; Sniegowski et al. 1997; Giraud et al. 2001; Shaver et al. 2002; de Visser and Rozen 2006). Similar findings have been reported for Saccharomyces cerevisiae (Thompson et al. 2006; Raynes et al. 2011, 2018). However, in contrast to findings in prokaryotes, mismatch-repair-deficient (henceforth $m m r$ ) or other types of strong mutators have not been found in natural $S$. cerevisiae populations (but see Bui et al. 2017; Raghavan et al. 2018), though weaker variation for the mutation rate has been detected (Gou et al. 2019). One explanation for this difference could be that $\mathrm{mmr}$ mutators experience higher load, compared with the wild type, in $S$. cerevisiae than they do in $E$. coli. Indeed, it has been observed by several investigators that haploid $\mathrm{mmr} S$. cerevisiae strains decline in frequency in the short term when co-cultured with wild-type strains (Thompson et al. 2006; Raynes et al. 2011, 2018; Bui et al. 2017), even if they eventually out-adapt the wild type. While this shortterm deficit of the fitness of $m m r$ mutators relative to the wild type has been attributed to increased mutational load in the $m m r$ strain, the evidence that this is the case has been mostly circumstantial (but see Wloch et al. 2001) because it is generally difficult to rule out an additional direct fitness effect of any allele thought to cause an indirect fitness effect (Raynes and Sniegowski 2014).

In this work, we establish, by short-term competitive fitness assays and in agreement with prior studies, that an $m m r$ haploid S. cerevisiae strain is substantially less fit than an otherwise isogenic $M M R+$ (i.e. wild-type) strain. This fitness difference could be caused solely by load, solely by some direct fitness effect of the $m m r$ phenotype, or some combination of the two. We develop separate assays to measure the components of load due to nonlethal and lethal deleterious mutations. To estimate the load caused by nonlethal deleterious mutations, we randomly sampled hundreds of clones from $\mathrm{mmr}$ and wild-type populations and measured the competitive fitness of each. The resulting histogram of the distribution of fitness of the $\mathrm{mmr}$ population provides an illustration of the effect of a high mutation rate on population mean fitness. We find that the means of these distributions differ, indicating substantial load for the $m m r$ strain, but not fully accounting for the total observed fitness difference between $\mathrm{mmr}$ and wild-type strains. To estimate the lethal mutation rate under the two different mutational regimes, we manipulate single cells to track the fate of mother/daughter duos. We show that these two separately measured components of load-due to nonlethal and lethal mutations-approximately sum to the measured fitness difference between the strains; hence we find no reason to suppose a direct fitness effect for $m m r$. Investigations with diploid versions of our strains provide support for this conclusion. We discuss some implications of these findings for continued experimental and theoretical investigations into the evolution of mutation rates.

\section{Materials and methods}

\section{Data analysis and figure production}

Data processing and analysis were performed in $\mathrm{R}$ (R Core Team 2019) and RStudio (RStudio Team 2015). Graphical output was produced using the package ggplot2 (Wickham 2016).

\section{Strains}

yJHK112, a haploid, prototrophic, heterothallic, MATa, BUD4-corrected, and ymCherry-labeled W303 strain was used as the haploid wild type in all work described in this paper. yJHK111, labeled with ymCitrine (a variant of YFP) and otherwise isogenic to yJHK112, was used as the "reference strain" in all haploid fitness competitions. These strains have been previously described (Koschwanez et al. 2013) and were generously provided by the laboratory of Andrew Murray, Harvard University, Cambridge, MA. An $m s h 2 \Delta$ derivative of yJHK112, in which the wild-type MSH2 allele was replaced with a kanMX geneticin (G418) resistance cassette (Wach et al. 1994), was used as the haploid $m m r$ mutator strain in all work described in this paper. This strain was generously provided by Yevgeniy Raynes of the laboratory of Dan Weinreich, Brown University, Providence, RI and has been previously described (Raynes et al. 2018). The kanMX cassette has been shown to not have a negative effect on growth (Baganz et al. 1997; Goldstein and McCusker 1999).

We constructed diploid versions of each of the three above strains by transforming (Gietz and Schiestl 2007) each with plasmid pRY003, temporarily providing a functional HO locus allowing mating type switching and subsequent mating. pRY003 was a gift from John McCusker 
(Addgene plasmid \#81043; http://n2t.net/addgene:81043; RRID:Addgene_81043). The diploid state of resulting isolates was confirmed by (1) ability to produce tetrads after plating to sporulation media; (2) by flow cytometry for total genomic content (following Gerstein et al. 2006); and (3) by the presence of a PCR product for both the MATa and $M A T \alpha$ loci. The $m m r$ diploids would not sporulate, but were confirmed to be diploids by the other two methods.

\section{Growth conditions}

The liquid medium for all fitness competitions was synthetic dextrose (SD) minimal medium containing yeast nitrogen base at a concentration of $6.7 \mathrm{~g} / \mathrm{L}$ and glucose at a concentration of $1.5 \mathrm{~g} / \mathrm{L}(0.15 \%)$, supplemented with tetracycline $(15 \mathrm{mg} / \mathrm{L})$ and ampicillin $(100 \mathrm{mg} / \mathrm{L})$. Fitness competitions were conducted in volumes of $200 \mu \mathrm{L}$ in deep polypropylene 96-well plates (Nunc 260251) sealed with flexible caps (Nunc 276002) and shaken at $1000 \mathrm{rpm}$ with an orbit of $3 \mathrm{~mm}$ (Corning LSE 6780-4) at a temperature of $30{ }^{\circ} \mathrm{C}$.

Initial growth in liquid for the lethal event assays was performed in SD as described above but without antibiotics, in flasks shaken at $200 \mathrm{rpm}$ at $30^{\circ} \mathrm{C}$. Growth on agar SD ( $2 \%$ glucose, no antibiotics) plates for the lethal assays took place at room temperature, $\sim 24^{\circ} \mathrm{C}$.

\section{Competitive fitness assays and isolation of clones}

Yeast, when grown by batch transfer with glucose as the carbon source, follow a relatively complex cycle of lag, fermentation, and respiration, and fitness benefits "accrued" in one phase (e.g. respiration) may not be "realized" until the next (e.g. the lag following the next transfer) (Li et al. 2018). Therefore we conducted short-term competitive fitness assays between wild-type and $m m r$ genotypes in which strains were mixed for one growth cycle prior to measuring frequencies (essentially, following Gallet et al. (2012)). The fitness assays were conducted as follows, with the interval between each consecutive day spanning $24 \mathrm{~h}$. Day 1: wildtype, $m m r$, and the $Y F P+$ reference strain were inoculated from frozen stock into single wells. Day 2: each strain was transferred to a new well with fresh medium, diluting 1/100. Day 3: competing strains were mixed $1: 1$ by volume and transferred to new wells with fresh medium, diluting $1 / 100$, to create 6 or 8 replicate competitions. Day 4: competitions were transferred to new wells with fresh medium, diluting $1 / 100$, and the frequencies of the competitor and reference strain were assayed by flow cytometry (Guava EasyCyte). Discrimination between strains was performed on the SSC/ GRN scatter plot. Day 5: the frequencies of the competitor and reference strain were again assayed by flow cytometry. The population density at the end of a 24-h cycle was $\sim 2 \times 10^{7}$ cells $/ \mathrm{mL}$; the census population size was thus $\sim 4 \times$ $10^{6}$ at transfer and $\sim 4 \times 10^{4}$ just after transfer.

The change in frequencies between Days 4 and 5 was used to calculate a selection coefficient $s$. Under a continuous model of growth (Crow and Kimura 1970, p. 193)

$s=\frac{1}{t} \ln \frac{p_{t}\left(1-p_{0}\right)}{p_{0}\left(1-p_{t}\right)}$,

from which a relative fitness $w=1+s$ follows. The number of generations, $t$, was assumed to be $\log _{2} 100$, or $\sim 6.64$. $p_{0}$ and $p_{t}$ are the starting and ending frequencies of the genotype being tested (i.e. the frequencies at Days 4 and 5 in the above procedure). The resulting selection coefficients represent differences in Malthusian parameter (that is, the $\log$ of Wrightian fitness) scaled per generation of growth.

We conducted fitness competitions using this procedure in eight separate blocks, each with multiple replicates as described above. Each block was begun on a different date. For each block, we computed the fitness of the $\mathrm{mmr}$ strain relative to the fitness of the wild-type strain by subtracting their mutual relative fitnesses to the reference strain. Each block included competitions in both haploid and diploid genotypes. Our final point estimate of the fitness difference between $m m r$ and wild-type strains is the mean difference across all blocks, and the 95\% confidence intervals (as shown in Fig. 2) were computed from the set of point estimates according to the $t$-distribution.

In two of the eight fitness competition blocks, we randomly sampled individual clones. To do so, we additionally propagated the haploid wild-type and $m m r$ strains on Day 3 in addition to mixing them 1:1 with the reference strain. Then, on Day 4, we plated these cultures, diluting appropriately, to YPD agar plates. After sufficient incubation, the resulting colonies were picked by pipet tip into wells containing $200 \mu \mathrm{L}$ YPD, grown for $24 \mathrm{~h}$, and frozen down by mixing 1:1 with $30 \%$ glycerol and storing at $-80{ }^{\circ} \mathrm{C}$ until needed for fitness assays. The random selection of colonies was ensured by either (1) picking all colonies on a given plate or (2) picking colonies concentrically from a randomly placed point.

Fitness assays for sets of isolated clones were conducted on a single 96-well plate, which allowed us to assay the fitnesses of 88 clones (some wells being reserved for various purposes) or fewer per run. We followed essentially the same procedure as the 5-day competition described above, except that frequencies were estimated at Day 3 and Day 4 instead of Day 4 and Day 5. This modification was made because some clones had such reduced fitnesses that an extra day of growth after mixing 1:1 with the reference strain caused the starting frequency of the clone to depart too greatly from $50 \%$.

The expected variance in measured selection coefficient due to random sampling effects during flow cytometry was 
1. Array microcolonies

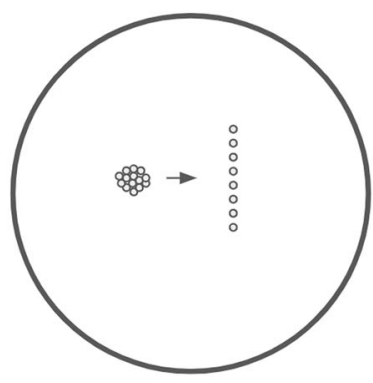

2. Separate new daughters

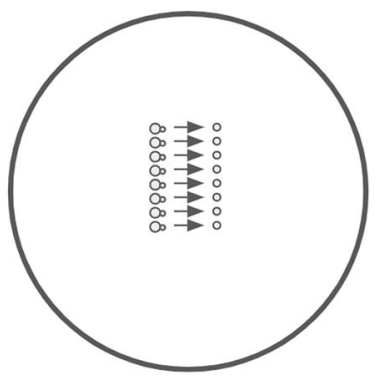

3. Score for lethal mutations

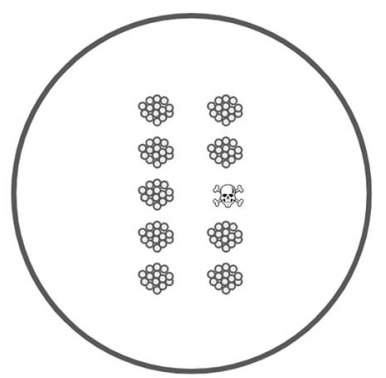

Fig. 1 Schematic of lethal assay. The arraying in step 1 and separation in step 2 were performed by micromanipulation. Microcolonies in step 1 comprised on average 23 cells, and were founded by new daughter cells that had themselves been isolated by micromanipulation

computed by means of a simple simulation in which the true frequency of each genotype at the start and end of the fitness competition was replaced by a random binomial variable. Ten thousand replicates of this simulation were run.

\section{Calculation of nonlethal load}

Mutational load is classically defined (Bürger 1998) as

$L=\frac{w_{\max }-\bar{w}}{w_{\max }}$,

where $\bar{w}$ is the mean fitness of the population and $w_{\max }$ is the fitness of the fittest genotype.

We measured all fitnesses relative to a common fluorescent strain, as described above. We define the unloaded fitness of each genotype as equal to 1 and we expect no beneficial mutations to rise to appreciable frequency in the short course of these experiments. Hence, $w_{\max }=1$ and thus

$L=1-\bar{w}$.

We expect measured selection coefficients to be distributed approximately normally around the true value, because of various sources of error including binomial sampling error, drift, instrument noise, environmental perturbations to individual wells (within-batch effects), and among 96-well plates (across-batch effects). To eliminate across-batch effects, for each run we adjusted all measured fitnesses by a constant $c$ such that the mode fitness is 1 (equivalently, such that the mode selection coefficient is zero). Once this adjustment has been made,

$L=1-\operatorname{mean}(W)$

where $W$ is the vector of all sampled clone fitnesses, or equivalently

$L=\operatorname{mean}(S)$ where $S$ is the vector of all sampled clone selection coefficients. A 95\% confidence interval for the nonlethal load was computed by bootstrapping from the measured fitnesses of all sampled clones: for 10,000 replicates, fitnesses were sampled randomly with replacement and the mean computed; from this empirical distribution, the 0.025 and 0.975 quantiles formed the bounds of the confidence interval.

\section{Lethal event assay}

Strains were inoculated from frozen stock into $6 \mathrm{~mL} \mathrm{SD} \mathrm{in} \mathrm{a}$ flask, and transferred to fresh media after $24 \mathrm{~h}$, diluting $1 / 100$. After an overnight of growth, a streak from the culture was made onto an SD agar plate and five single cells with nascent buds were physically isolated by means of a Zeiss (West Germany) micromanipulating microscope fitted with a Singer Instruments (Somerset, UK) dissecting needle. These cells were periodically checked over the next few hours and the daughters physically separated once developed. These daughters became the founders of microcolonies that were allowed to grow at room temperature for $\sim 20 \mathrm{~h}$, reaching an average size of 23.3 cells (22.8 for the wild type, 23.8 for $m m r$, difference not significant at $p>$ $0.6)$. These microcolonies were then dissected into a gridlike arrangement of single cells (step 1 in Fig. 1). These cells were then checked at intervals of $1-2 \mathrm{~h}$ and daughters separated as soon as possible (step 2 in Fig. 1). The colonies resulting from these mother/daughter duos were checked at $\sim 24, \sim 48$, and in some cases $\sim 72$ and $\sim 96 \mathrm{~h}$ after separation. A lethal event was recorded when the growth of a mother or daughter lineage ceased. In such cases cessation of growth was sometimes immediate and sometimes occurred after a few generations. In the latter cases the growth was generally markedly slowed by the first observation. In a few cases, slow but unceasing growth was noted: these are presumed to be cases in which a strongly deleterious mutation 
occurred, though we stress that this assay was not designed to detect nonlethal deleterious mutations.

As described in the "Discussion" section, the difference in the rate of lethal events between the wild type and $\mathrm{mmr}$ strains was used as the estimate for the lethal mutation rate for the $m m r$ strain. A $95 \%$ confidence interval for this difference in rates was computed by the prop.test function in $\mathrm{R}$ (Newcombe 1998).

\section{Fluctuation assays}

To measure the mutation rate to 5 -fluoroorotic acid (5-FOA) resistance, we employed the following procedure. Strains of interest were inoculated into $10 \mathrm{~mL}$ YPD, grown in flasks with shaking at $30^{\circ} \mathrm{C}$ for $\sim 24 \mathrm{~h}$, and then transferred to $30 \mathrm{~mL}$ fresh YPD diluting such that $\sim 200$ cells were passaged, in replicates of 5 . After $\sim 48 \mathrm{~h}$ of growth, each replicate was plated without dilution to $\mathrm{SD}+5$-FOA $(1 \mathrm{~g} / \mathrm{L})$ agar plates to estimate density and absolute number of resistants, and plated with a $10^{-5}$ dilution to YPD agar plates to estimate total population density and absolute number. Plates were counted after $\sim 48 \mathrm{~h}$ of growth and mutation rates were estimated using the maximum likelihood method of Gerrish (2008). For each round of fluctuation tests, we estimated mutation rates for both wild-type and $m m r$ strains simultaneously in order to minimize the influence of any uncontrolled sources of variation.

\section{Homopolymers per gene}

The per-base rate of homopolymeric runs of various lengths in $S$. cerevisiae coding regions were computed by a custom Python script. The S. cerevisiae S288C reference genome was downloaded from www.yeastgenome.org.

\section{Results}

\section{Mutation rate elevation in $\mathbf{m m r}$ strain}

To confirm that the $m m r(m s h 2 \Delta)$ strain is a mutator, we conducted fluctuation tests using resistance to 5-FOA as the selectable phenotype. Averaged across replicate fluctuation tests, we found a 20.8 -fold increase (95\% CI: 13.4 - to $28.3-$ fold) in the mutation rate for the $\mathrm{mmr}$ strain relative to the wild type (Fig. S1). This is likely an underestimate of the effective genome-wide increase in the mutation rate because $m m r$ mutators have a greatly elevated indel rate for homopolymeric runs (Lang et al. 2013), of which URA3, the main locus involved in this fluctuation test, is relatively devoid (Fig. S4).

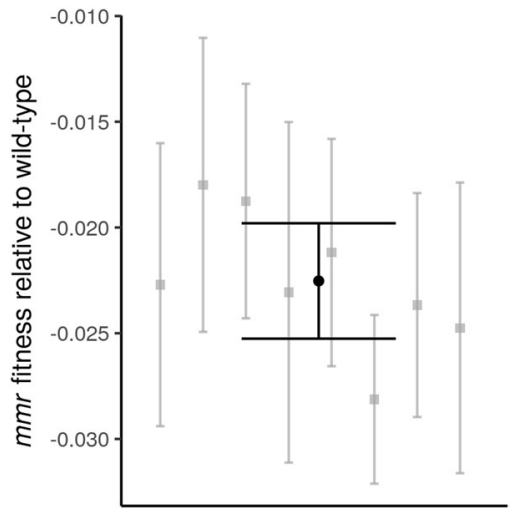

Fig. 2 The average competitive fitness deficit (in black, error bars are $95 \%$ confidence intervals) of the mutator strain relative to the wild type, expressed as selection coefficient, is $2.3 \%$. Fitness competitions were conducted in a series of eight blocks, shown in gray. The two strains were not competed directly against each other; within each block, each was competed separately against an otherwise isogenic $M M R+Y F P+$ strain. Each block contained between 6 and 8 replicate competitions

\section{Fitness disadvantage of $\mathbf{m m r}$ compared with wild- type}

We competed $m m r$ and wild-type strains against a common YFP + reference strain. Averaged across eight separate blocks of fitness competitions, we found the mutator to be less fit than the wild-type, with an average fitness deficit, expressed as a selection coefficient per generation, of $\sim 2.25 \%$ (95\% CI: $1.98-2.53 \%$ ) (Fig. 2).

\section{Estimation of nonlethal load}

We randomly sampled individual clones from both $m m r$ and wild-type populations and measured the competitive fitness of each clone. The sampled fitness distributions are shown in Fig. 3. The $m m r$ strain's fitness distribution has a prominent left tail of less-fit individuals. We calculated the load as the difference between the mode and the mean fitness; this is $\sim 0$ for the wild-type strain and $\sim 1.65 \%$ (95\% CI: $2.19-1.08 \%)$ for the $m m r$ strain. The difference in load between the two strains is significant $\left(p<10^{-8}\right)$. The fitness distributions for the $m m r$ and wild type are significantly different in shape (Kolmogorov-Smirnov test; $p<10^{-8}$ ), while the fitness distribution for the wild-type strain is not significantly different from a normal distribution with the same mean and variance (Kolmogorov-Smirnov test; $p>0.05)$. 
A

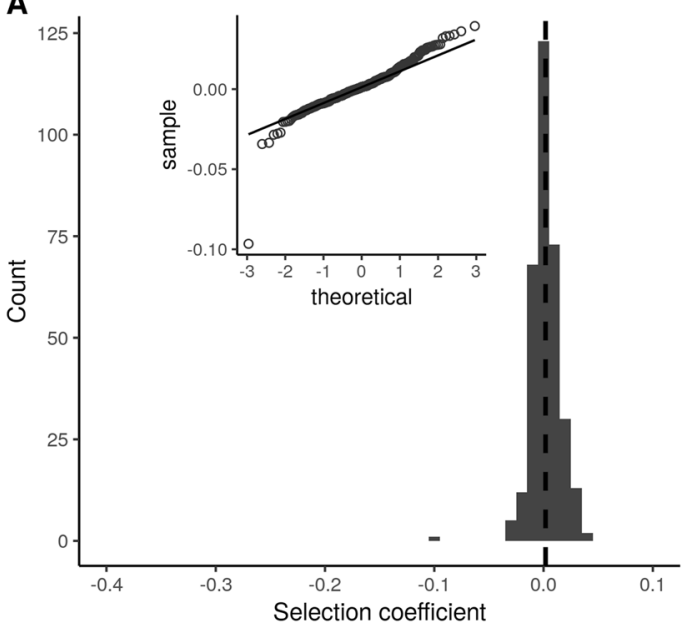

Fig. 3 Distributions of fitness in haploid wild types (a) and $\mathrm{mmr}$ mutators (b). We measured the fitnesses of 327 wild type and $313 \mathrm{mmr}$ clones. Fitnesses were measured in competition with an $M M R+Y F P+$ reference strain otherwise isogenic to the wild type, as described in

\section{Estimation of lethal mutation rate}

To assay lethal mutation rates, we manipulated single $S$. cerevisiae cells, separating mother/daughter duos and tracking events in which one member of the duo failed to found a colony. The procedure is shown in Fig. 1 and explicated more fully in the "Methods" section. Assaying over 2200 duos for each strain, we found a rate of lethal events per newly replicated genome of $0.31 \%$ (95\% CI: $0.018-0.055 \%$ ) in the wild-type strain and $0.76 \%$ (95\% CI: $0.53-1.07 \%$ ) in the $m m r$ strain (Table 1). The difference between these rates is $0.44 \%$ (95\% CI: $0.12 \%-0.77 \%$ ). Because the observed rate of lethal events in the wild type is much higher than the expected lethal mutation rate, we take this difference as our estimate of the lethal mutation rate in the $m m r$ strain (see the "Discussion" section for elaboration on this point). Photographs of representative lethal events are shown in Fig. S3.

In our assay we followed separated duos that contained a suspected lethal until growth ceased. In some cases growth never ceased, but doubling times were very slow compared with the usual growth rate; such cases were not counted as lethal events but are tallied separately in Table 1. We also detected cases in which both members of a duo were lethal, or both showed strongly reduced growth, and also cases in which the mother cell never divided. Because such events probably stemmed from a mutation that occurred prior to the division that created the duo under observation, we excluded these events from our analysis.

\section{Results in diploids}

From the haploid strains, we constructed diploid $m m r$ and wild-type strains. We calculate the nonlethal load in the

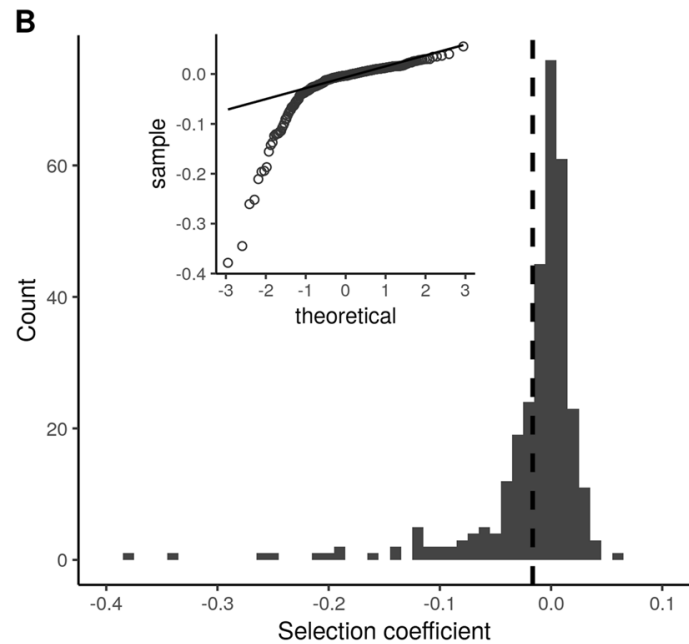

"Methods" section. Dashed vertical lines indicate the mean. The load is $\sim 0$ for wild types; for $m m r$ mutators it is $\sim 1.7 \%$. QQ plots of the fitness distributions are shown as insets. The two distributions differ significantly (Kolmogorov-Smirnov test, $p<10^{-8}$ )

Table 1 Counts and frequencies of events of interest in the lethal assay.

\begin{tabular}{llllllll}
\hline \multirow{2}{*}{ Event } & \multicolumn{2}{l}{ Wild type } & & & $m m r$ & \\
\cline { 2 - 3 } & Count & Rate & & Count & Rate & & $p$-value \\
\hline One lethal & 2235 & 0.9967 & 2145 & 0.9908 & 0.0006 \\
$\begin{array}{l}\text { One strongly } \\
\text { reduced growth }\end{array}$ & 14 & 0.0031 & 33 & 0.0076 & 0.0049 \\
& 1 & 0.0002 & 7 & 0.0016 & 0.0363 \\
& & & & & & \\
\hline
\end{tabular}

"One lethal" means that the lineage founded by either the mother or daughter cell ceased to grow within the observation period. "One strongly reduced growth" means that either the mother or daughter lineage was observed to grow noticeably slowly. Other events-both members of the duo lethal or strongly reduced growth, or the mother never budding-were not included in the analysis and are not displayed here. The $p$-values reflect the statistical significance of the difference in rates between wild type and $m m r$ strains and were obtained by Fisher's exact test. Note that events are displayed per duo while rates are calculated per individual. "Both OK" means that both mother and daughter cell grew into normal colonies

$m m r$ diploid strain as $\sim 0$ for the wild-type diploid strain and $0.30 \%$ (95\% CI: -0.02 to $0.55 \%$ ) (Fig. S2) for the $m m r$ strain-substantially less, by about $80 \%$, than the equivalent load in the $m m r$ haploid strain (difference significant at $p<10^{-4}$ ). The diploid wild-type and $m m r$ distributions differ significantly in shape (Kolmogorov-Smirnov test; $p$ $\left.<10^{-4}\right)$ but do not differ significantly in mean $(p>0.05)$.

We also measured the difference in population fitness between wild-type and $m m r$ diploid strains via short-term competitive fitness assays. We found that the $m m r$ diploid is less fit than the wild-type diploid by a selection coefficient of $\sim 1.69 \%$ (95\% CI: $1.40-1.94 \%$ ) (Fig. 4). This difference, though larger than expected, is smaller than the fitness 


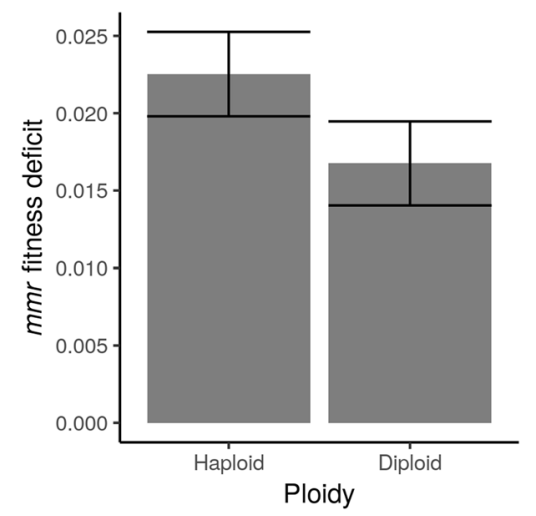

Fig. 4 Change in $m m r$ fitness disadvantage with ploidy. The fitness deficit, relative to the wild type, is $26 \%$ lower in diploids than in haploids $(p<0.004)$

difference between wild-type and $m m r$ haploid strains by $26 \%$ (haploid-to-diploid difference significant at $p<0.004$ ).

\section{Discussion}

Prior work has shown that, over the short term, haploid $\mathrm{mmr}$ $S$. cerevisiae strains decline in frequency when competed with a strain that is wild-type for the mutation rate (Thompson et al. 2006; Raynes et al. 2011, 2018; Bui et al. 2017). Consistent with these findings, we find a fitness disadvantage, expressed as a selection coefficient per generation, of $\sim 2.3 \%$ for $\mathrm{mmr}$ haploids in short-term fitness competitions (Fig. 2). The magnitude of this selective disadvantage is similar to that in other reports, including Raynes et al. (2011) (2.4\% cost), Raynes et al. (2018) (3.3\% cost), and Wloch et al. (2001) (4.6\% cost, though this is a noncompetitive measure of absolute growth rate).

The deleterious mutations that cause load include both lethal and nonlethal mutations. There is no fundamental theoretical distinction between these two classes of mutation insofar as their contribution to load is concerned: in many population genetic models, all members of an asexual population who are not of the least-loaded class are considered to be doomed (Rice 2002). However, their different manifestations require different experimental techniques. We therefore developed separate approaches to measure these two components of load.

\section{Load due to nonlethal mutations}

We measured the short-term competitive fitnesses of 640 randomly selected haploid clones. The histogram and QQ plot for the haploid wild-type populations (Fig. 3a) suggest that, apart from one less-fit clone, the distribution of fitness for the wild-type strain is essentially normal. The normality of the distribution is consistent with nearly all wild-type clones having the same genotype and thus the same expected fitness, along with many small sources of error in estimation of fitness. One such source of error is drift over the course of the short-term fitness competition. The formula derived by Gallet et al. (2012) suggests that the expected variance in fitness measurement due to drift given our experimental parameters is $\sim 2 \times 10^{-6}$. A larger source of variance is due to sampling error: in the fitness competitions, we estimate the relative frequencies of the competitors at two time points, sampling $~ 8000$ cells per time point. We carried out simulations that suggest that the expected variance due to sampling error is $\sim 2.3 \times 10^{-5}$. These two sources of variance, summed, make up about $20 \%$ of the observed variance in selection coefficient. The remainder of the variance probably stems from small-scale environmental variation and other unknown sources of error.

In contrast to the results in wild types, the fitness histogram and QQ plot for the haploid $m m r$ strain (Fig. 3b) are not reflective of a normal distribution. Instead, a prominent left tail of less-fit clones demonstrates the effect of mutational load. The mean selection coefficient is approximately $-1.7 \%$, which is the quantification of the reduction in population mean fitness due to nonlethal load. This reduction accounts for a substantial portion $(\sim 75 \%)$ of the measured competitive fitness difference (Fig. 2) between the two strains.

Our estimate of the nonlethal load $(\sim 1.7 \%)$ reflects the average per-generation growth deficit of the mutator subpopulation due to the accumulation of deleterious mutations up to the point of random sampling of clones. We note that this is an estimate of the load at a nonequilibrium state, and is expected to be less than the full load achieved when mutation-selection balance is reached for all loci. Direct observation of mutation-selection equilibrium in a laboratory setting would be challenging because experimental populations rapidly generate adaptive mutations. Our strainto-strain fitness assays, which found (Fig. 2) a fitness deficit of $2.3 \%$ for the $m m r$ strain relative to the wild type, are likewise reflective of a nonequilibrium state. Since both estimates are derived from the same nonequilibrium populations, they are directly comparable.

Selection coefficients of about the magnitude we observe here cause changes in relative frequency that are extremely rapid in evolutionary terms. For example, a selective deficit of $2 \%$ would cause a decline from $50 \%$ to $20 \%$ frequency in 70 generations. Observing a rapid initial decline of haploid mmr S. cerevisiae strains in competition with wild types, some investigators (e.g. Grimberg and Zeyl 2005) have attributed the observed fitness difference to an unknown 
direct cost (i.e. a pleiotropic effect) while others (e.g. Raynes et al. 2018) have assumed that mutational load fully explains the dynamics. The question has remained open, in part because it has been nearly impossible to definitively rule out a direct fitness effect of being $\mathrm{mmr}$ - any attempt to measure such an effect will be confounded by the indirect fitness effects. By quantifying the indirect fitness effects (i.e. load) we seek to determine if a direct effect need be invoked to explain the observed experimental dynamics.

It is not surprising that the nonlethal load accounts for only a portion of the observed fitness difference. The nonlethal load assay relies on the growth of deleterious mutants in order to measure their fitness and thus cannot detect mutants that do not grow, i.e. lethal mutations. In order to measure this portion of the load, we designed an assay in which lethal events are directly observed.

\section{Load due to lethal mutations}

The lethal mutation rate has long been a matter of interest (e.g. Dobzhansky and Wright 1941). By observing 4435 mother-daughter pairs (duos), we found a rate of lethal events of 0.0076 and 0.0031 for the $m m r$ and wild-type strain, respectively.

Our observed wild-type lethal event rate, 0.0031, is on the order of estimates for the genomic mutation rate itself (Drake 1991; Lynch et al. 2008; Zhu et al. 2014; Sharp et al. 2018) and therefore cannot plausibly reflect the rate of lethal mutations. Our interpretation is that, for the wild type, all or most observed lethal events were not caused by genomic mutations and are instead best considered to be nonmutational deaths, perhaps caused by fine-scale environmental fluctuations, experimental manipulation, or other stochastic sources of insult and stress. Observations of relatively high rates of cell death, too high to be due to lethal mutation, are not uncommon. Replicative aging studies of $S$. cerevisiae often observe low but substantial rates of cell death even in very young mother cells (e.g. Chiocchetti et al. 2007; Shcheprova et al. 2008). Rates of cell death on the order of our observed rate for the wildtype strain have also been observed in young bacterial cells (Wang et al. 2010), suggesting that relatively high rates of nonmutational, non-age-related deaths are common among microbes. Our assay design ensured that colonies were young (the oldest cell in a microcolony was on average $\sim 4.3$ generations old) and we did not observe a bias in lethal events towards mothers (Table S1), so we do not attribute the observed lethal events to senescence. In fact, we observed, across both strains, a bias towards the lethal event occurring in the daughter cell. This difference was not statistically significant $(p=0.14)$, although within the $m m r$ strain only we observed 10 lethal events in mothers and 23 in daughters $(p=0.04)$. The observed bias towards daughters dying, if not a sampling effect, could be attributable to smaller daughter cells being relatively more vulnerable to stress. Indeed, increased vulnerability of daughters to environmental sources of stress has been previously reported (Knorre et al. 2010).

An a priori estimation of the wild-type lethal mutation rate can be made as follows. Lang and Murray (2008) conducted careful estimations of the rate of loss-of-function mutations to the CAN1 locus in a similar background (W303) as the strains used in this work. Multiplying this rate, $1.5 \times 10^{-7}$, by the number of genes thought to be essential for viability, $\sim 1100$ (Giaever et al. 2002), and accounting for the fact that $C A N 1$ is longer than the average essential gene gives an expected lethal rate in wild-type haploids of $1.5 \times 10^{-4}$. This estimate is on the upper end but within the range of observed rates of accumulation of recessive lethals in several experiments conducted with diploids (Wloch et al. 2001; Hall and Joseph 2010; Nishant et al. 2010; Zhu et al. 2014; Jasmin and Lenormand 2016). Such a rate would suggest that we expected to observe about 0.3 lethal mutations in the wild-type strain in our experiment; we actually observed 14 . Therefore, we consider the observed rate of lethal events in the wild type to be an estimate of the rate of nonmutational deaths. The corresponding rate for the $m m r$ strain is 0.0076 (difference significant at $p<0.001$ ). Making the assumption that nonmutational deaths equally affect both strains, we take the difference between the wild-type and $m m r$ lethal event rates, 0.0044 (95\% CI: 0.0012-0.0077), as the estimate of the lethal mutation rate in the $\mathrm{mmr}$ strain. We note that our empirical result is fairly close to the figure obtained by multiplying the wild-type a priori estimate, $1.5 \times 10^{-4}$, by the average fold increase in CAN1 loss-of-function mutation rate for $m m r$ strains in a collection of published reports (44fold; see Table S4). A slightly different methodology, taking the average CANl loss-of-function rate of $\mathrm{mmr}$ strains from published reports $\left(1.5 \times 10^{-5}\right.$; Table S4) and multiplying by 1100 essential genes yields a somewhat higher expected lethal mutation rate of $\sim 0.015$.

In many of the lethal events that we observed, growth did not immediately cease but continued for a few generations (Table S2) before halting. Limited growth for a few generations after an ultimately lethal mutation occurs has previously been observed (Mortimer 1955). We also observed morphological defects in several lethal events; one such instance is shown in the bottom panel of Fig. S3. We note that some lethal mutations that we observed could be due to chromosomal losses during mitosis (aneuploidies), but that knocking out $M S H 2$ has not been observed to greatly increase the rate of such events in haploids (Serero et al. 2014). 


\section{Diploid findings}

We measured the short-term competitive fitnesses of 573 randomly selected diploid clones. The distribution of fitness for $m m r$ diploids (Fig. S2) suggests that they are substantially less loaded than $m m r$ haploids, as would be expected if dominance attenuates the deleterious effects of new mutations. We calculate the nonlethal load in $\mathrm{mmr}$ diploids as $\sim 0.3 \%$, as opposed to $\sim 1.7 \%$ in $\mathrm{mmr}$ haploids: that is, $\sim 80 \%$ of the load has gone away following diploidization. One interpretation of this finding is that deleterious mutations tend to be recessive in diploids. Thus, comparison of the fitness distributions of $\mathrm{mmr}$ diploids and $\mathrm{mmr}$ haploids is consistent with a high mutuation rate and diploidy shielding the effects of deleterious mutations.

The sampled wild-type diploid clones included more low-fitness individuals than the wild-type haploids (compare Fig. S2A with Fig. 3a). We cannot fully explain this observation; one possible explanation is that diploids are more prone than haploids to nondisjuctions causing aneuploid chromosomes, a notion for which there is some experimental support (Sharp et al. 2018).

The relative difference in short-term competitive fitness between wild-type and $\mathrm{mmr}$ strains is narrowed by $26 \%$ in diploids (Fig. 4). It is somewhat surprising, given that the nonlethal loads are not very different between wild-type and $m m r$ diploids, that this figure is not larger. One possibility is that the diploid mutator fixed a deleterious mutation during the process of diploidization, which would account for the discrepancy between the reduction in nonlethal load (82\%) and the reduction in total fitness difference (26\%) in diploids compared with haploids. Another formal possibility is that diploid mutators have a higher lethal mutation rate than haploid mutators, but we cannot posit a causative mechanism for such an effect.

\section{Considering the two loads together}

The total fitness difference between the haploid wild-type and $m m r$ strains could be a consequence of greater mutational load for the $m m r$ strain, a direct effect of the $m s h 2 \Delta$ deletion, or a combination of the two. The addition of the lethal and nonlethal loads $(0.0166+0.0044=0.0210)$ is $\sim 7 \%$ smaller than the measured fitness difference $(0.0225)$, and the difference is not significant (Fig. S5). The difference may simply be due to sampling error, or due to systematic underestimation of one or the other of the loads. The nonlethal load may be slightly underestimated because clones were isolated by plating at the beginning of the growth cycle during which competitive fitness was measured. The load may have continued to increase somewhat during this growth cycle.
The broad equivalence of the sum of the loads, on the one hand, and the strain-to-strain competitive fitness, on the other, is consistent with the hypothesis that the total fitness difference is solely due to mutational load. Hence, although we cannot rule out the existence of a small direct fitness effect, these findings suggest that there is no need to invoke direct effects in explaining the fitness difference between the $m m r$ and wild-type haploid strains.

The load is equal to the deleterious mutation rate only when the population is in mutation-selection balance. This equilibrium is reached instantly for lethal mutations, quickly for deleterious mutations of large effect, and very slowly for deleterious mutations of slight effect (Johnson 1999). The $m m r$ populations in our assays experienced, including the initial process of transformation and growth before frozen storage, about 60 generations of growth, which is enough time to achieve mutation-selection balance for deleterious mutations of relatively large effect, but not for deleterious mutations of slight effect. Hence, our estimate of the total load (2.1-2.3\%) should be considered an estimate of the lower limit for the deleterious mutation rate for $\mathrm{mmr}$ haploids.

\section{Comparison with results in bacteria}

Insofar as $S$. cerevisiae and E. coli are two model organisms, from different domains of life, with which many evolution experiments have been performed, it is interesting to compare the loads of mismatch repair mutators in both. It appears that in $E$. coli the relative fitness deficit for $m m r$ strains is smaller than it is in haploid $S$. cerevisiae. For instance, Shaver et al. (2002) did not detect a fitness difference between $m m r$ and wild-type strains, de Visser and Rozen (2006) did not observe an initial decline in mutS frequency when that genotype was competed with the wild type at different starting ratios, and Boe et al. (2000) estimated at most a $1 \%$ selective disadvantage for $\mathrm{mmr}$ mutators. In this context it is relevant to note that there are several reports of $m m r$ genotypes in natural $E$. coli isolates (LeClerc et al. 1996; Matic et al. 1997; Denamur et al. 2002), as well as in other types of bacteria (Oliver et al. 2000; Richardson et al. 2002; Trong et al. 2005; Gould et al. 2007). In S. cerevisiae, in contrast, no functionally $\mathrm{mmr}$ natural isolates have yet been found (though see Raghavan et al. 2018). Such observations suggest that E. coli may be relatively more robust than $S$. cerevisiae to the lack of a functional mismatch repair system. One reason for this difference could be that the genomic mutation rate in $E$. coli is lower than that of $S$. cerevisiae by a factor of about 4 (Lee et al. 2012, Lynch et al. (2008); Zhu et al. 2014; Sharp et al. 2018). If there is a similar absolute difference in deleterious mutation rate, then even if the relative 
fold increase in the deleterious mutation rate caused by the lack of mismatch repair is also equal in both organisms, the absolute difference in load, which is what controls the evolutionary dynamics, will be larger in $S$. cerevisiae than in E. coli. Another possible factor is differences in the spectrum and genomic substrate of mutations. In both $E$. coli and $S$. cerevisiae, the indel rate is greatly increased in $m m r$ lineages, and the rate of indels is strongly elevated in homopolymeric repeats (HPRs). Both the relative increase from wild-type to $\mathrm{mmr}$ and the absolute indel rate in $\mathrm{mmr}$ are higher, and scale upwards faster with HPR length, in $S$. cerevisiae than in E. coli (Schaaper and Dunn 1991; Tran et al. 1997; Gragg et al. 2002; Lee et al. 2012; Lang et al. 2013). Examining all coding sequences in the E. coli and $S$. cerevisiae genomes, we find that there are significantly more HPRs per coding genome, per gene, and per coding base in S. cerevisiae than in E. coli (Table S5). S. cerevisiae that are $\mathrm{mmr}$ are therefore relatively more burdened by indels than are $m m r$ E. coli which could account for both the apparent larger fitness difference between $M M R+$ and $m m r$ and the corresponding apparent contrast in occurrence in natural isolates. We caution that this particular analysis is speculative in nature at this time: one important caveat is that, while this study and others have found large fitness differences between wild-type and $m m r$ haploids, $S$. cerevisiae spend most of their time in nature as diploids, in which the fitness deficit of $m m r$ lineages might be less severe: classically, equilibrium mutational load is halved in the recessive case compared with the additive, or haploid, case (Kimura et al. 1963). However, while estimates of the rate of outcrossing in S. cerevisiae are very low (Ruderfer et al. 2006), the rate of sporulation, which entails a haploid stage, is not known, and evidence of extensive inbreeding and loss of heterozygosity (Peter et al. 2018) suggest that it is relatively frequent. Recessive deleterious mutations may thus be frequently exposed to selection in natural $S$. cerevisiae populations by both the haploid life cycle stage and loss of heterozygosity from inbreeding, suggesting that diploidy may not be as much of a shield for $m m r$ lineages as it otherwise would be. A second caveat is that, even if there is no direct fitness effect of $m m r$ in haploids, there could be such an effect in diploids, perhaps due to misregulation of the frequency of recombination events (reviewed in Surtees et al. 2004; George and Alani 2012).

\section{Conclusions and future directions}

We have found that the indirect fitness effects of strong modifiers for mutation rates are substantial in haploid $S$. cerevisiae, and that it is not necessary to postulate direct fitness effects in order to explain the selective disadvantage of the lack of a functional mismatch repair pathway. This finding is probably most relevant to experimental inquiries of the dynamics of mutation rate evolution in which $S$. cerevisiae is the model organism.

We have also reported findings relevant to fundamental questions about mutational dynamics, including the lethal mutation rate and the relative ratio of lethal and nonlethal deleterious mutations. By sampling the fitnesses of many individuals we have clearly demonstrated mutational load in an $m m r$ population, and from the load we are able to estimate a lower limit for the deleterious mutation rate. We sampled hundreds of clones and were able to obtain a clear picture of the left tail of the fitness distribution for the $m m r$ strain, but not for the wild-type strain. If the fitnesses of tens of thousands of clones could be measured, much could be learned about load and other evolutionary dynamics at wildtype mutation rates; such experiments may become possible as methods for high-throughput measurements continue to advance.

A limitation of this study is that we captured a snapshot of mutational load at a particular point in time in an evolving population. It would be interesting to observe, at a fine scale, how the distribution of fitnesses changes over time as a population approaches mutation-selection balance, adapts, and experiences other population genetic processes.

Acknowledgements This research was facilitated by a National Aeronautics and Space Administration grant (NNA15BB04A) to PDS and a National Science Foundation Graduate Research Fellowship to BGS. We thank the laboratories of Andrew Murray (Harvard University, Cambridge, MA) and Dan Weinreich (Brown University, Providence, RI) for their generous sharing of yeast strains.

Data availability Data available from the Dryad Digital Repository: https://doi.org/10.5061/dryad.4b10j7q.

\section{Compliance with ethical standards}

Conflict of interest The authors declare that they have no conflict of interest.

Publisher's note Springer Nature remains neutral with regard to jurisdictional claims in published maps and institutional affiliations.

\section{References}

Baganz F, Hayes A, Marren D, Gardner DCJ, Oliver SG (1997) Suitability of replacement markers for functional analysis studies in Saccharomyces cerevisiae. Yeast 13:1563-1573

Boe L, Danielsen M, Knudsen S, Petersen JB, Maymann J, Jensen PR (2000) The frequency of mutators in populations of Escherichia coli. Mutat Res 448:47-55

Bui DT, Friedrich A, Al-Sweel N, Liti G, Schacherer J, Aquadro CF et al. (2017) Mismatch repair incompatibilities in diverse yeast populations. Genetics 205:1459-1471

Bürger R (1998) Mathematical properties of mutation-selection models. Genetica 102:279

Chao L, Cox EC (1983) Competition between high and low mutating strains of Escherichia coli. Evolution 37:125 
Charlesworth B (2015) Causes of natural variation in fitness: evidence from studies of Drosophila populations. PNAS 112:1662-1669

Chiocchetti A, Zhou J, Zhu H, Karl T, Haubenreisser O, Rinnerthaler M et al. (2007) Ribosomal proteins Rpl10 and Rps6 are potent regulators of yeast replicative life span. Exp Gerontol 42:275-286

Cox EC, Gibson TC (1974) Selection for high mutation rates in chemostats. Genetics 77:169-184

Crow JF, Kimura M (1970) An introduction to population genetics theory. Harper \& Row, New York, NY

Danforth CH (1923) Eugenics, genetics and the family. In: Scientific papers of the Second International Congress of Eugenics, American Museum of Natural History, New York, 22-28 Sept 1921. Williams \& Wilkins Company.

de Visser JAGM, Rozen DE (2006) Clonal interference and the periodic selection of new beneficial mutations in Escherichia coli. Genetics 172:2093-2100

Denamur E, Matic I (2006) Evolution of mutation rates in bacteria. Mol Microbiol 60:820-827

Denamur E, Bonacorsi S, Giraud A, Duriez P, Hilali F, Amorin C et al. (2002) High frequency of mutator strains among human uropathogenic Escherichia coli isolates. J Bacteriol 184:605-609

Desai MM, Fisher DS (2011) The balance between mutators and nonmutators in asexual populations. Genetics 188:997-1014

Dobzhansky T, Wright S (1941) Genetics of natural populations. V. Relations between mutation rate and accumulation of lethals in populations of Drosophila pseudoobscura. Genetics 26:23

Drake JW (1991) A constant rate of spontaneous mutation in DNAbased microbes. Proc Natl Acad Sci USA 88: 7160-7164

Fisher R (1930) The genetical theory of natural selection. Clarendon Press, Oxford

Gallet R, Cooper TF, Elena SF, Lenormand T (2012) Measuring selection coefficients below $10^{-3}$ : method, questions, and prospects. Genetics 190:175-186

George CM, Alani E (2012) Multiple cellular mechanisms prevent chromosomal rearrangements involving repetitive DNA. Crit Rev Biochem Mol Biol 47:297-313

Gerrish P (2008) A simple formula for obtaining markedly improved mutation rate estimates. Genetics 180:1773-1778

Gerstein AC, Chun H-JE, Grant A, Otto SP (2006) Genomic convergence toward diploidy in Saccharomyces cerevisiae. PLOS Genet 2:e145

Giaever G, Chu AM, Ni L, Connelly C, Riles L, Véronneau S et al. (2002) Functional profiling of the Saccharomyces cerevisiae genome. Nature 418:387-391

Gietz R, Schiestl R (2007) Quick and easy yeast transformation using the LiAc/SS carrier DNA/PEG method. Nat Protoc 2:35-7

Giraud A, Matic I, Tenaillon O, Clara A, Radman M, Fons M et al. (2001) Costs and benefits of high mutation rates: adaptive evolution of bacteria in the mouse gut. Science 291:2606-2608

Goldstein AL, McCusker JH (1999) Three new dominant drug resistance cassettes for gene disruption in Saccharomyces cerevisiae. Yeast 15:1541-1553

Gou L, Bloom JS, Kruglyak L (2019) The genetic basis of mutation rate variation in yeast. Genetics 211:731-740

Gould CV, Sniegowski PD, Shchepetov M, Metlay JP, Weiser JN (2007) Identifying mutator phenotypes among fluoroquinoloneresistant strains of Streptococcus pneumoniae using fluctuation analysis. Antimicrob Agents Chemother 51:3225-3229

Gragg H, Harfe BD, Jinks-Robertson S (2002) Base composition of mononucleotide runs affects DNA polymerase slippage and removal of frameshift intermediates by mismatch repair in Saccharomyces cerevisiae. Mol Cell Biol 22:8756-8762

Grimberg B, Zeyl C (2005) The effects of sex and mutation rate on adaptation in test tubes and to mouse hosts by Saccharomyces cerevisiae. Evolution 59:431-438
Gross MD, Siegel EC (1981) Incidence of mutator strains in Escherichia coli and coliforms in nature. Mutat Res Lett 91:107-110

Haldane JBS (1937) The effect of variation of fitness. Am Nat 71:337-349

Hall DW, Joseph SB (2010) A high frequency of beneficial mutations across multiple fitness components in Saccharomyces cerevisiae. Genetics 185:1397-1409

Jasmin J-N, Lenormand T (2016) Accelerating mutational load is not due to synergistic epistasis or mutator alleles in mutation accumulation lines of yeast. Genetics 202:751-763

Johnson T (1999) The approach to mutation-selection balance in an infinite asexual population, and the evolution of mutation rates. Proc Biol Sci 266: 2389-2397

Jyssum K (1960) Observations on two types of genetic instability in Escherichia coli. Acta Pathol Microbiol Scand 48:113-120

Kimura M, Maruyama T, Crow JF (1963) The mutation load in small populations. Genetics 48:1303-1312

Knorre DA, Kulemzina IA, Sorokin MI, Kochmak SA, Bocharova NA, Sokolov SS et al. (2010) Sir2-dependent daughter-to-mother transport of the damaged proteins in yeast is required to prevent high stress sensitivity of the daughters. Cell Cycle 9:4501-4505

Koschwanez JH, Foster KR, Murray AW (2013) Improved use of a public good selects for the evolution of undifferentiated multicellularity. eLife 2:e00367

Lang GI, Murray AW (2008) Estimating the per-base-pair mutation rate in the yeast Saccharomyces cerevisiae. Genetics 178:67-82

Lang GI, Parsons L, Gammie AE (2013) Mutation rates, spectra, and genome-wide distribution of spontaneous mutations in mismatch repair deficient yeast. G3: Genes, Genomes. Genetics 3:1453-1465

LeClerc JE, Li B, Payne WL, Cebula TA (1996) High mutation frequencies among Escherichia coli and Salmonella pathogens. Science 274:1208-1211

Lee H, Popodi E, Tang H, Foster PL (2012) Rate and molecular spectrum of spontaneous mutations in the bacterium Escherichia coli as determined by whole-genome sequencing. Proce Natl Acad Sci USA 109: E2774-E2783

Li Y, Venkataram S, Agarwala A, Dunn B, Petrov DA, Sherlock G et al. (2018) Hidden complexity of yeast adaptation under simple evolutionary conditions. Curr Biol 28:515-525.e6

Lynch M, Sung W, Morris K, Coffey N, Landry CR, Dopman EB et al. (2008) A genome-wide view of the spectrum of spontaneous mutations in yeast. PNAS 105:9272-9277

Matic I, Radman M, Taddei F, Picard B, Doit C, Bingen E et al. (1997) Highly variable mutation rates in commensal and pathogenic Escherichia coli. Science 277:1833-1834

Mortimer RK (1955) Evidence for two types of x-ray-induced lethal damage in Saccharomyces cerevisiae. Radiat Res 2:361-368

Muller HJ (1950) Our load of mutations. Am J Hum Genet 2:111-176

Newcombe RG (1998) Interval estimation for the difference between independent proportions: comparison of eleven methods. Stat Med 17:873-890

Nishant KT, Wei W, Mancera E, Argueso JL, Schlattl A, Delhomme N et al. (2010) The baker's yeast diploid genome is remarkably stable in vegetative growth and meiosis. PLOS Genet 6:e1001109

Oliver A, Cantón R, Campo P, Baquero F, Blázquez J (2000) High frequency of hypermutable Pseudomonas aeruginosa in cystic fibrosis lung infection. Science 288:1251-1253

Peter J, Chiara MD, Friedrich A, Yue J-X, Pflieger D, Bergström A et al. (2018) Genome evolution across 1,011 Saccharomyces cerevisiae isolates. Nature 556:339

R Core Team (2019) R: a language and environment for statistical computing. R Foundation for Statistical Computing, Vienna, Austria

Raghavan V, Bui DT, Al-Sweel N, Friedrich A, Schacherer J, Aquadro $\mathrm{CF}$ et al. (2018) Incompatibilities in mismatch repair genes 
MLH1-PMS1 contribute to a wide range of mutation rates in human isolates of baker's yeast. Genetics 210:1253-1266

Raynes Y, Sniegowski PD (2014) Experimental evolution and the dynamics of genomic mutation rate modifiers. Heredity 113:375-380

Raynes Y, Gazzara MR, Sniegowski PD (2011) Mutator dynamics in sexual and asexual experimental populations of yeast. BMC Evol Biol 11:158

Raynes Y, Wylie CS, Sniegowski PD, Weinreich DM (2018) Sign of selection on mutation rate modifiers depends on population size. PNAS 115:3422-3427

Rice WR (2002) Experimental tests of the adaptive significance of sexual recombination. Nat Rev Genet 3:241

Richardson AR, Yu Z, Popovic T, Stojiljkovic I (2002) Mutator clones of Neisseria meningitidis in epidemic serogroup A disease. PNAS 99:6103-6107

RStudio Team (2015) RStudio: integrated development environment for R. RStudio, Inc., Boston, MA

Ruderfer DM, Pratt SC, Seidel HS, Kruglyak L (2006) Population genomic analysis of outcrossing and recombination in yeast. Nat Genet 38:1077-1081

Schaaper RM, Dunn RL (1991) Spontaneous mutation in the Escherichia coli lacI gene. Genetics 129:317-326

Serero A, Jubin C, Loeillet S, Legoix-Né P, Nicolas AG (2014) Mutational landscape of yeast mutator strains. PNAS 111:1897-1902

Sharp NP, Sandell L, James CG, Otto SP (2018) The genome-wide rate and spectrum of spontaneous mutations differ between haploid and diploid yeast. PNAS 115:E5046-E5055

Shaver AC, Dombrowski PG, Sweeney JY, Treis T, Zappala RM, Sniegowski PD (2002) Fitness evolution and the rise of mutator alleles in experimental Escherichia coli populations. Genetics 162:557-566
Shcheprova Z, Baldi S, Frei SB, Gonnet G, Barral Y (2008) A mechanism for asymmetric segregation of age during yeast budding. Nature 454:728-734

Sniegowski PD, Gerrish PJ, Lenski RE (1997) Evolution of high mutation rates in experimental populations of E. coli. Nature 387:703-705

Surtees JA, Argueso JL, Alani E (2004) Mismatch repair proteins: key regulators of genetic recombination. Cytogenet Genome Res 107:146-159

Thompson DA, Desai MM, Murray AW (2006) Ploidy controls the success of mutators and nature of mutations during budding yeast evolution. Curr Biol 16:1581-1590

Tran HT, Keen JD, Kricker M, Resnick MA, Gordenin DA (1997) Hypermutability of homonucleotide runs in mismatch repair and DNA polymerase proofreading yeast mutants. Mol Cell Biol 17:2859-2865

Trong HN, Prunier A-L, Leclercq R (2005) Hypermutable and fluoroquinolone-resistant clinical isolates of Staphylococcus aureus. Antimicrob Agents Chemother 49:2098-2101

Wach A, Brachat A, Pöhlmann R, Philippsen P (1994) New heterologous modules for classical or PCR-based gene disruptions in Saccharomyces cerevisiae. Yeast 10:1793-1808

Wang P, Robert L, Pelletier J, Dang WL, Taddei F, Wright A et al. (2010) Robust growth of Escherichia coli. Curr Biol 20:1099-1103

Wickham H (2016) Ggplot2: elegant graphics for data analysis. Springer, Houston, Texas

Wloch DM, Szafraniec K, Borts RH, Korona R (2001) Direct estimate of the mutation rate and the distribution of fitness effects in the yeast Saccharomyces cerevisiae. Genetics 159:441-452

Zhu YO, Siegal ML, Hall DW, Petrov DA (2014) Precise estimates of mutation rate and spectrum in yeast. Proc Natl Acad Sci USA 111: E2310-E2318 